\section{(2) OPEN ACCESS}

\title{
Outcomes in patients with a first episode of chest pain undergoing early coronary $\mathrm{CT}$ imaging
}

\author{
Klaske R Siegersma (1) , ${ }^{1,2} \mathrm{~N}$ Charlotte Onland-Moret, ${ }^{3}$ Yolande Appelman, ${ }^{1}$ \\ Pim van der Harst, ${ }^{4}$ Igor I Tulevski, ${ }^{5} \mathrm{G}$ Aernout Somsen, ${ }^{5}$ Jagat Narula, ${ }_{1}^{6}$ \\ Hester M den Ruijter (i) ${ }^{2}$ Leonard Hofstra ${ }^{1,5}$
}

- Additional supplemental material is published online only. To view, please visit the journal online (http://dx.doi. org/10.1136/heartjnl-2021319747).

'Department of Cardiology, Amsterdam UMC VUMC Site Amsterdam, The Netherlands ${ }^{2}$ Laboratory of Experimental Cardiology, University Medical Center Utrecht, Utrecht University, Utrecht, The Netherlands

${ }^{3}$ Julius Center for Health Sciences and Primary Care, University Medical Center Utrecht, Utrecht University, Utrecht, The Netherlands ${ }^{4}$ Department of Cardiology, University Medical Center Utrecht, Utrecht University, Utrecht, The Netherlands ${ }^{5}$ Cardiology Centers of the Netherlands, Utrecht, The Netherlands

${ }^{6}$ Icahn School of Medicine at Mount Sinai, New York, New York, USA

\section{Correspondence to}

Professor Leonard Hofstra, Cardiology Centers of the Netherlands, Utrecht, 3584 AA, The Netherlands; leonard.hofstra@gmail.com

Received 22 May 2021 Accepted 11 October 2021

Check for updates

(c) Author(s) (or their employer(s)) 2021. Re-use permitted under CC BY-NC. No commercial re-use. See rights and permissions. Published by BMJ.

To cite: Siegersma KR Onland-Moret NC,

Appelman Y, et al. Heart Epub ahead of print:

[please include Day Month

Year]. doi:10.1136/

heartjnl-2021-319747

\begin{abstract}
Objectives To investigate the impact of a CT-first strategy on all-cause and cardiovascular mortality in patients presenting with chest pain in outpatient cardiology clinics.
\end{abstract}

Methods Patients with a first presentation of suspected angina pectoris were identified and their data linked to the registrations of Statistics Netherlands for information on mortality. The linked database consisted of 33068 patients. CT-first patients were defined as patients with a CT calcium score and coronary CT angiography, within 6 weeks after their initial visit. Propensity score matching (1:5) was used to match patients with and without a CT-first strategy. After matching, 12545 patients were included of which 2308 CT-first patients and 10237 patients that underwent usual care.

Results Mean age was 57 years, $56.3 \%$ were women and median follow-up was 4.9 years. All-cause mortality was significantly lower in CT-first patients ( $n=43,1.9 \%$ ) compared with patients without CT $(n=363,3.5 \%)$ (HR: $0.51,95 \% \mathrm{Cl} 0.37$ to 0.70). Furthermore, CT-first patients were more likely to receive cardiovascular preventative and antianginal medication (aspirin: 44.9\% vs $27.1 \%$, statins: $48.7 \%$ vs $30.3 \%$, beta-blockers: $37.8 \%$ vs $25.5 \%$, in CT-first and without CT-first patients, respectively) and to undergo downstream diagnostics and interventions (coronary interventions: $8.5 \%$ vs $5.7 \%$, coronary angiography: $16.2 \%$ vs $10.6 \%$ in CT-first and without CT-first patients, respectively). Conclusions In a real-world regular care database, a CT-first strategy in patients suspected of angina pectoris was associated with a lowering of all-cause mortality.

\section{INTRODUCTION}

Longitudinal studies of employing cardiac CT imaging including both coronary calcium scoring (CACS) and cardiac CT angiography (CCTA) in patients presenting with chest pain have demonstrated incremental prognostic value compared with traditional risk profiling algorithms. ${ }^{1}$ Higher CACS is associated with mortality ${ }^{2}$ and has added value to the Framingham Risk Score for predicting cardiovascular events. ${ }^{4}$ The introduction of CCTA showed even greater promise as a tool to define risk of myocardial infarction ${ }^{5}$ and coronary revascularisation ${ }^{6}$ compared with CACS. Prospectively randomised trials showed that CCTA was superior to functional cardiac testing for cardiovascular endpoints. $^{78}$
Until the results of the SCOT-HEART (Scottish COmputed Tomography of the HEART Trial) study were published, ${ }^{5}$ CCTA was only adopted by the guidelines of the National Institute for Health and Care Excellence in the UK. ${ }^{9}$ The SCOT-HEART study randomised patients presenting with chest pain in the cardiology outpatient clinic to either CCTA initiated, the so-called CT-first strategy, or routine clinical care. Patients randomised to the CCTA arm showed only about half of the fatal and non-fatal myocardial infarctions after a 5-year follow-up, ${ }^{5}$ although these results were not in line with the previously published PROMISE-trial ${ }^{8}$ and multiple registry studies. ${ }^{10} 11$ Nevertheless, these results prompted the European Society of Cardiology to accord CCTA a class I recommendation for the diagnosis of coronary artery disease (CAD) in symptomatic patients in the renewed European Society of Cardiology (ESC) guidelines of 2019. ${ }^{12}$

However, the results of SCOT-HEART might not be generalisable to a routine care population who are expected to have a higher likelihood of mortality, since randomised trials may induce an overestimation of benefit due to the so-called healthy volunteer inclusion bias in trials. ${ }^{13} 14$ Furthermore, multiple patient groups are often under-represented in clinical studies, such as women, ${ }^{15}$ the elderly, non-white ethnicities and patients with comorbidities. ${ }^{1617}$ Therefore, the impact of a CT-first strategy for patients with chest pain in regular care is still unknown. ${ }^{1011}$

The use of data from real-world databases to assess the utility of cardiac CT may aid in defining associations between patient variables and outcomes in the general population. Moreover, real-world data may help to vindicate results of clinical trials, especially with the inclusion of substantial numbers of all relevant patient groups. Thus, we used a regular care database ${ }^{18}$ to investigate all-cause and cardiovascular mortality in patients suspected of angina pectoris, who underwent CT calcium score and CCTA following their first visit, and compared this with a propensity score (PS) matched control group from the same database, who were subjected to regular care without cardiac CT.

\section{METHODS}

\section{Study population}

The CCN database consists of 109151 patients who visited one of the CCN's outpatient cardiology clinics between 2007 and 2018. These centres 


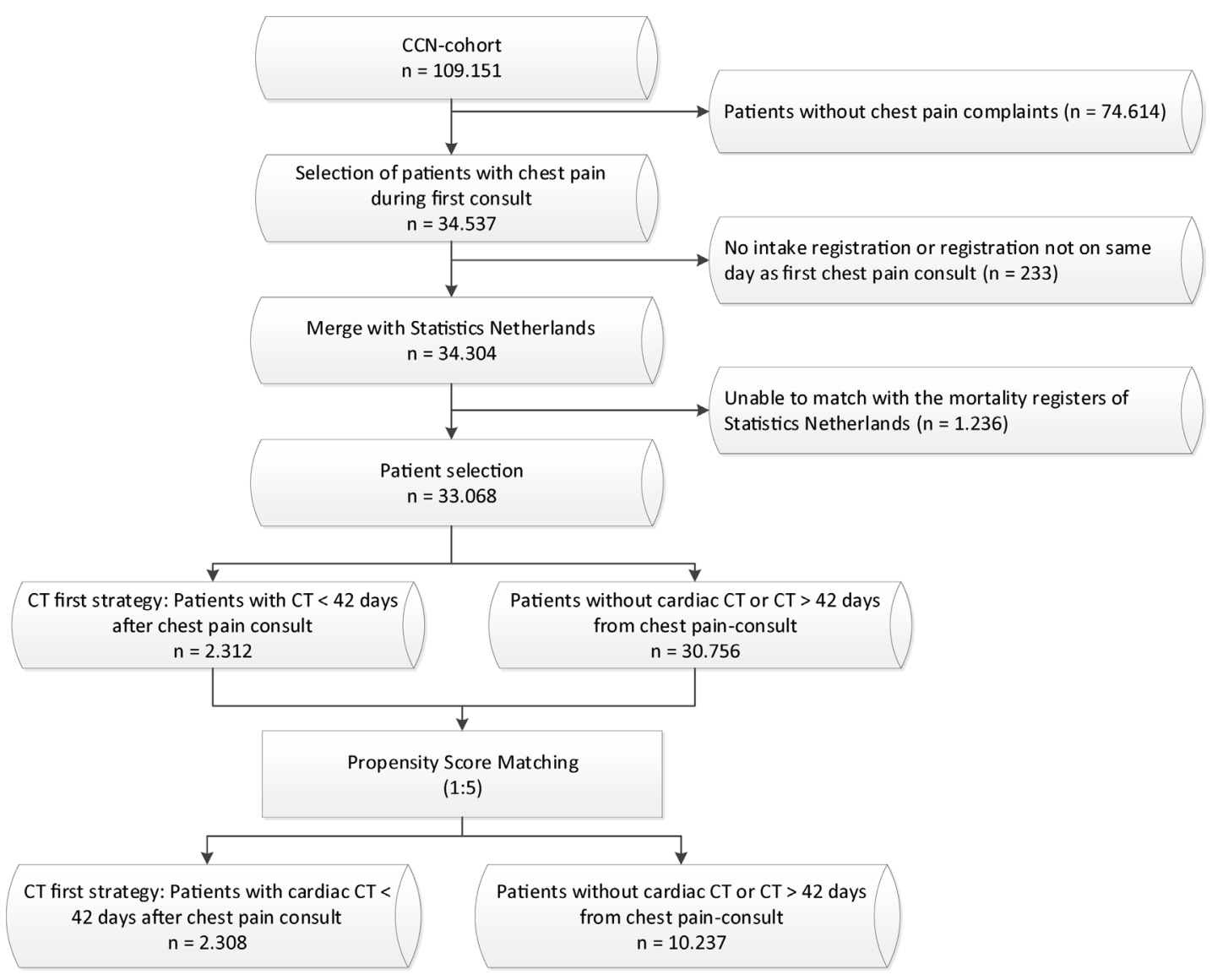

Figure 1 Flow chart of patient selection. CCN, Cardiology Centers of the Netherlands.

are known for their homogenised and structured approach to investigate patients with cardiovascular complaints. A detailed description of the CCN database has been published. ${ }^{18}$ Patients with chest pain suspected to be angina pectoris, who presented for the first time at one of the diagnostic centres, were included. The study population was then split in two groups: patients with a cardiac CT within 6 weeks after their first visit, the CT-first strategy, and patients without this diagnostic procedure. Cardiac CT included a scanning protocol for calcium scoring and CCTA. The decision for referral for cardiac CT was made by the treating cardiologist. The type of CT scanner was based on availability in the referenced centres. Follow-up of the population was obtained through linking with the population database of Statistics Netherlands (CBS). Figure 1 outlines the selection of the study population.

The Cardiology Centers of the Netherlands data were made available under implied consent and transferred to the University Medical Center Utrecht under the Dutch Personal Data Protection Act. This study used data collected during the regular care process and did not subject participants to additional procedures or impose behavioural patterns on them. The Medical Research Ethics Committee of the University Medical Center Utrecht declared that research within the CCN database does not meet the Dutch Medical Research Involving Human Subjects Act (proposal number 17/359).

\section{Study variables}

Patient characteristics, prior comorbidities, risk factors, family history and a general medical history were obtained from the electronic health records (EHRs) of the patient. Medication use was extracted from pharmaceutical prescription data. Residential region of the patient was obtained through four-digit postal code. Chest pain was characterised as typical, atypical or non-anginal, according to Diamond ${ }^{1219}$ and obtained through retrieval of text variables in the EHR. We also included the results from the stress ECG recording to ensure comparability between patients with and without a CT-first strategy.

\section{Short-term and long-term outcome}

All-cause mortality and cardiovascular mortality were the primary and secondary outcome in the analyses, respectively. Other secondary outcomes included registration of diagnostic and therapeutic procedures in the EHR. Diagnostics included stress ECG recording, functional imaging (cardiac MRI, positron emission tomography and single-photon emission computed tomography), invasive coronary angiography (CAG) and cardiac CT more than 6 weeks after the first visit to CCN. Therapeutic procedures were percutaneous coronary intervention and coronary artery bypass grafting. Moreover, prescribed medication changes during and after the chest pain consult were evaluated and compared between the groups. This analysis focused primarily on the value of a cardiac CT in the diagnostic trajectory of the patient. We did not evaluate the association between results of cardiac CT and primary and secondary outcomes.

\section{Missing data}

Missing data in variables required for analysis were handled by multiple imputation for chained equations (MICE; 10 iterations, 10 imputed datasets) with the R-package mice (V.3.8.0). ${ }^{20}$ However, for the presence of a family history, missing data were not at random. Therefore, missing values for this variable 


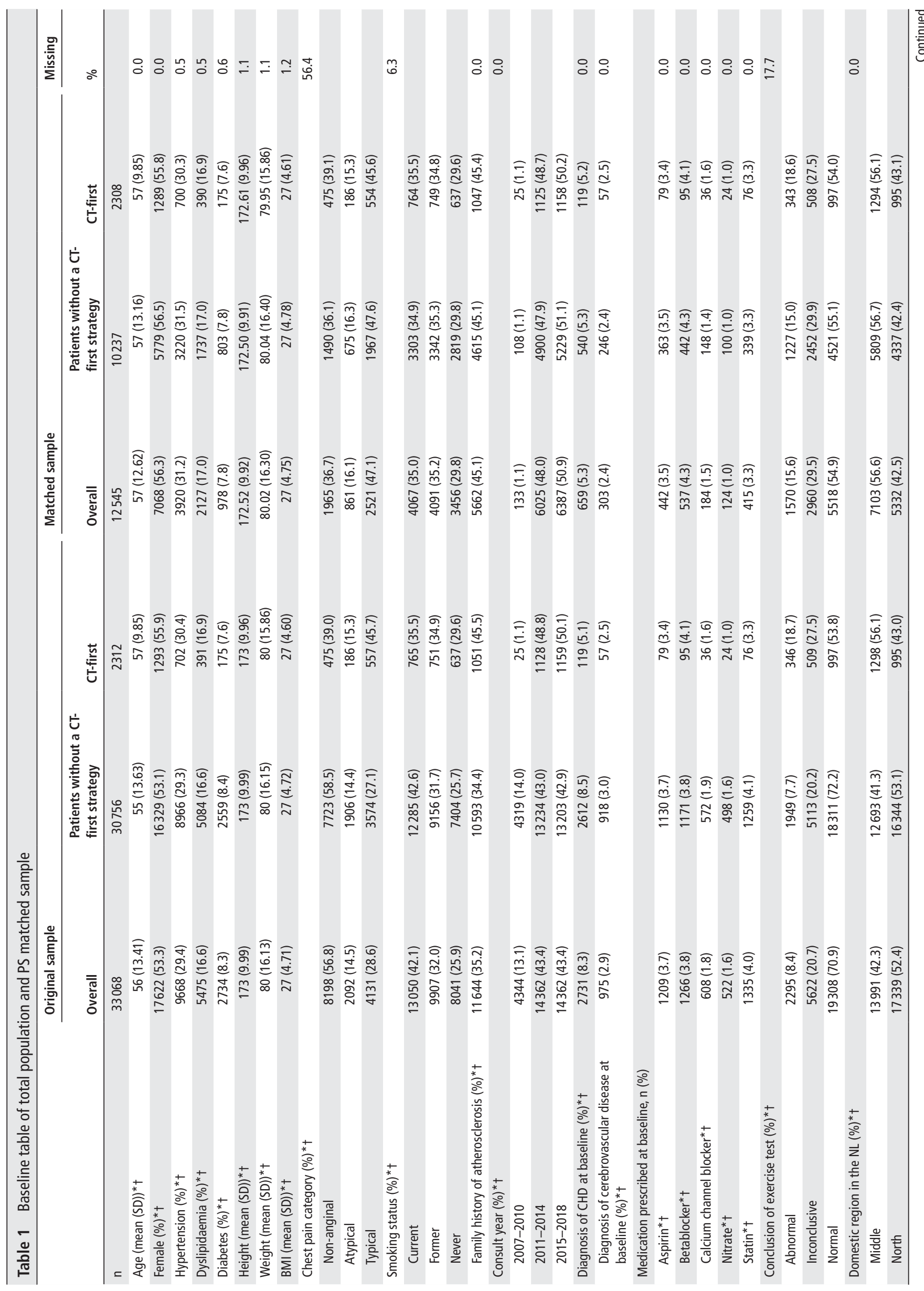




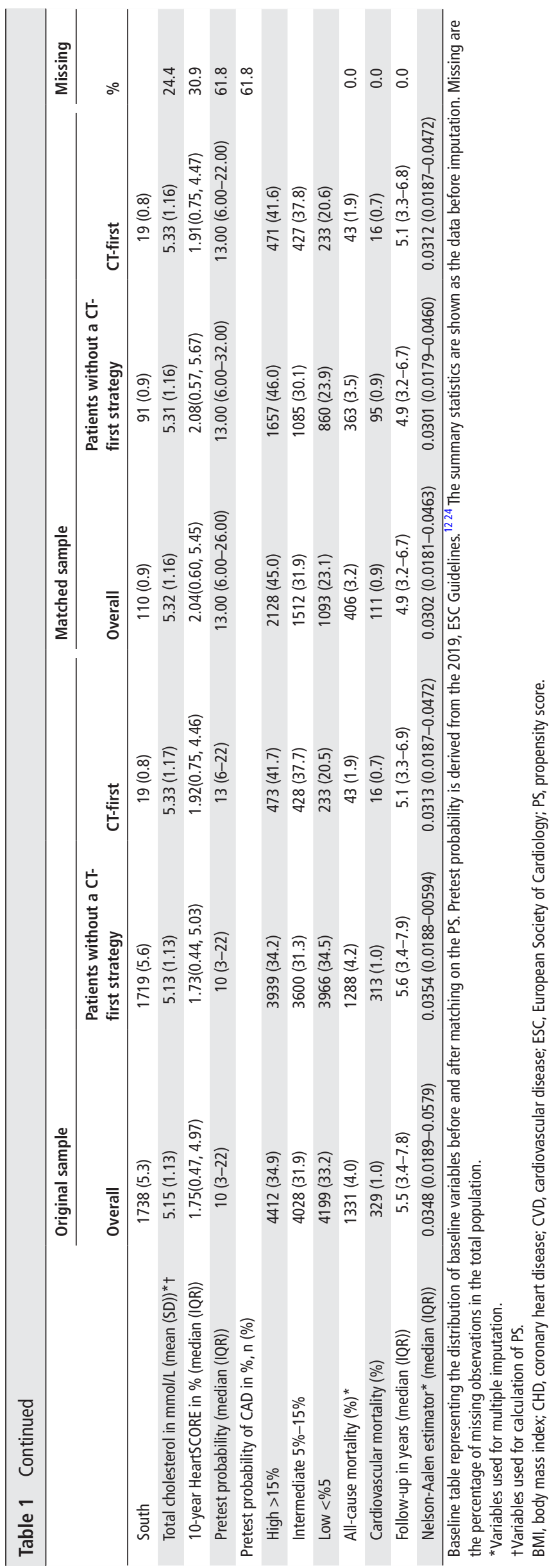

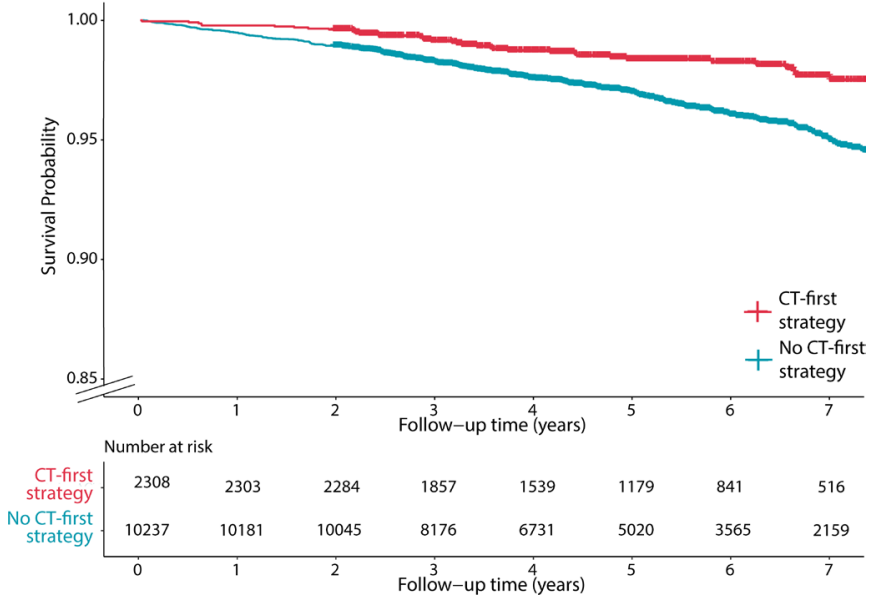

Figure 2 Kaplan-Meier curves for all-cause mortality of the CT-first and the without a CT-first study population.

were filled with a negative family history. Included variables for multiple imputation models are indicated in table 1.

\section{PS matching}

PS matching ${ }^{21}$ was used to ensure a comparable sample between patients with and without a CT-first strategy. PSs were calculated for each patient separately in each imputed dataset with a logistic regression model including 19 variables, as indicated in table 1 . This selection of variables was based on clinically relevant variables and baseline differences between both groups. Thereafter, the average PS of the imputation datasets was calculated for each patient. We matched five patients who did not receive a CT-first strategy to one patient with a CT-first strategy based on the calculated PSs per patient using the nearest neighbour method with a calliper width of 0.05 and no replacement (R-package: matchit, ${ }^{22}$ V.3.0.2). Comparability of the groups after matching was assessed by inspection of the balance of baseline variables. The selected patient population is further referred to as the matched sample.

\section{Statistical analysis}

Descriptive statistics are presented as mean with SD or median with IQR, where appropriate, for continuous variables and counts and percentages for categorical variables. All-cause and cardiovascular mortality were analysed with Kaplan-Meier curves and Cox regression models on the matched patient selection. Other secondary outcomes were compared with $\chi^{2}$ testing.

Subgroup analyses were performed for type of chest pain (anginal/non-anginal), sex (men/women), age ( $<65$ years $/ \geq 65$ years), Systematic COronary Risk Evaluation (SCORE) $(<5 \% / \geq 5 \%)^{23}$ and pretest probability of CAD $(<5 \% />5 \%){ }^{24}$ The $\mathrm{p}$ value for interaction was determined for each analysis. Outcome for all subgroup analyses was all-cause mortality.

Sensitivity analyses were done to evaluate the effect of patient's residency and inclusion year. In these analyses, a Cox regression for all-cause mortality was performed. Another sensitivity analysis excluded all patients referred to one specific diagnostic centre, only performing CCTA in case of medical need or if calcium score was above 0 . As no information of the centre that was visited is available in the database, residency of the patient is used as a substitute. A final sensitivity analysis removed all patients without a CT but with CAG to evaluate the effect on the 
results. These sensitivity analyses evaluated Cox regressions for both cardiovascular and all-cause mortality.

All data analyses were done with $\mathrm{R}$ (V.3.6.2) and RStudio (V.1.1.463).

\section{Patient and public involvement}

Patients were not involved in any stage of this research process.

\section{RESULTS}

\section{Study population}

A total of 34311 patients with chest pain met the inclusion criteria and were selected from the CCN database (figure 1). After linking with the database of Statistics Netherlands, mortality data of 33068 (96.4\%) patients were available. This selection included 2312 and 30756 patients, respectively, with and without a CT-first strategy.

Mean age of the included patients $(n=33068)$ was 56 years and $53.3 \%$ were women. Before PS matching, patients in the CT-first group (table 1 ) were older (57 vs 55 years), less likely to be current smokers (35.5\% vs $42.6 \%)$ and had a higher incidence of typical chest pain $(45.7 \%$ vs $27.1 \%)$. Yet, the presence of comorbidities was comparable. Median follow-up was 5.5 (IQR: 3.4-7.8) years, and 1331 (4.0\%) patients died during follow-up. In $329(1.0 \%)$ patients, a cardiovascular cause of death was assigned. Thus, PSs were distributed differently between patients with and without a CT-first strategy (online supplemental figure $1)$.

After PS matching, 2308 patients were in the CT-first group, and 10237 patients were matched to this group. Patients in the CT-first group had a median coronary artery calcification score of 2.20 (IQR: 0-88). There were 871, 329 and 225 filed stenoses in the left anterior descending artery, right coronary artery and circumflex artery, respectively, of which 489 (34.3\%) were reported to be significant ( $>50 \%$ stenosis). In the matched sample, baseline characteristics were more equally distributed as compared with the unmatched sample (table 1), for example, mean age (57 years in both groups) and type of chest pain (non-anginal $39.1 \%$ vs $36.1 \%$, atypical $15.3 \%$ vs $16.3 \%$, and typical $45.6 \%$ vs $47.6 \%$ for, respectively, with and without a CT-first strategy). Consequently, the distribution of the PS of the included patients showed improved overlap in the matched sample (online supplemental figure 1). The matched sample $(n=12545)$ was used for the analyses of primary and secondary outcomes.

\section{Outcome analysis}

All-cause and cardiovascular mortality after PS matching

In the matched sample 406 (3.2\%) patients died. In 111 (0.9\%) patients, there was a cardiovascular cause of death. Median follow-up was 4.9 years. In patients with a CT-first strategy, all-cause mortality was $1.9 \%(n=43)$ compared with $3.5 \%$ $(\mathrm{n}=363)$ in patients without a CT-first strategy (HR: 0.51, 95\% CI 0.37 to 0.70). The Kaplan-Meier curves for patients with and without a CT-first strategy are shown in figure 2 and show a consistent divergent pattern over up to 7 years of follow-up. Cardiovascular mortality for patients in the CT-first group was $16(0.7 \%)$ and $95(0.9 \%)$ for the patients without a CT-first strategy, respectively. The corresponding HR for cardiovascular mortality was 0.73 (95\% CI 0.43 to 1.24). The corresponding Kaplan-Meier curves are shown in online supplemental figure 2 .

\section{Downstream diagnostics and interventions}

A total of $3432(27.4 \%)$ patients of the matched cohort had diagnostic or therapeutic follow-up. The percentage of CAGs $(16.2 \%$ vs $10.6 \%, \mathrm{p}<0.001)$ and coronary interventions $(8.5 \%$ vs $5.7 \%, \mathrm{p}<0.001$ ) were higher in the CT-first strategy group, compared with the group of patients without CT (table 2). The same was seen for the number of stress ECGs, which was higher for CT-first patients $(17.5 \%$ vs $15.6 \%, p=0.03)$, yet the time interval between initial presentation and stress ECG was shorter in patients without a CT-first strategy (median time between chest pain consult and stress ECG: 112 days, IQR: 25-598, vs 301 days, IQR: 91-759). Follow-up cardiac perfusion imaging did not show any differences $(3.0 \%$ vs $2.8 \%$ in, respectively, patients with and without a CT-first strategy).

\section{Medication use}

Medication use for primary or secondary prevention of CAD (including aspirin, statins, beta-blockers, calcium channel blockers and nitrates) at baseline was similar for patients with and without a CT-first strategy (tables 1 and 3). After initial chest pain consult, initiation of aspirin (44.9\% vs $27.1 \%, \mathrm{p}<0.001)$, b-blockers $(37.8 \%$ vs $25.5 \%, \mathrm{p}<0.001)$ and statins $(48.7 \%$ vs $30.3 \%, \mathrm{p}<0.001)$ was higher in the CT-first strategy group, compared with the group without CT.

Table 2 Distribution of diagnostics and therapeutics during follow-up

\begin{tabular}{|c|c|c|c|}
\hline & $\begin{array}{l}\text { CT-first } \\
(n=2308)\end{array}$ & $\begin{array}{l}\text { Patients without a CT-first strategy } \\
(\mathrm{n}=10237)\end{array}$ & $P$ value \\
\hline Anatomical imaging (\%) & $392(17.0)$ & $1435(14.0)$ & $<0.001$ \\
\hline Perfusion imaging (\%) & $70(3.0)$ & $290(2.8)$ & 0.652 \\
\hline Coronary interventions (\%) & $197(8.5)$ & $581(5.7)$ & $<0.001$ \\
\hline Stress ECG at CCN (\%) & $403(17.5)$ & $1600(15.6)$ & 0.032 \\
\hline Coronary angiography (\%) & $373(16.2)$ & $1086(10.6)$ & $<0.001$ \\
\hline Cardiac CT (\%) & $23(1.0)$ & $429(4.2)$ & $<0.001$ \\
\hline Time (days) between chest pain consult - stress ECG (median (IQR)) & $301.00(91.00-759.00)$ & $112.00(25.00-597.75)$ & $<0.001$ \\
\hline Time (days) between chest pain consult - anatomical imaging (median (IQR)) & $57.00(37.75-87.25)$ & $49.00(20.00-119.50)$ & $<0.001$ \\
\hline Time (days) between chest pain consult - perfusion imaging (median (IQR)) & $74.00(49.25-162.25)$ & $36.50(17.00-91.00)$ & $<0.001$ \\
\hline Time (days) between chest pain consult - coronary intervention (median (IQR)) & $65.00(42.00-98.00)$ & $51.00(21.00-102.00)$ & $<0.001$ \\
\hline
\end{tabular}

Distribution of diagnostics and therapeutics during follow-up in patients with a CT-first strategy and the population without a CT-first strategy. Anatomical imaging comprises cardiac CT and coronary angiography. Perfusion imaging includes cardiac PET, SPECT and MRI. Coronary interventions are coronary artery bypass grafts and percutaneous coronary interventions.

CCN, Cardiology Centers of the Netherlands; MRI, magnetic resonance imaging; PET, positron emission tomography; SPECT, single-photon emission computed tomography. 
Table 3 Medication use in selected population

\begin{tabular}{|c|c|c|}
\hline & $\begin{array}{l}\text { CT-first } \\
(n=2308)\end{array}$ & $\begin{array}{l}\text { Patients without a CT-first strategy } \\
(\mathrm{n}=10237)\end{array}$ \\
\hline \multicolumn{3}{|l|}{ Aspirin (\%) } \\
\hline Continued & $57(2.5)$ & $323(3.2)$ \\
\hline Discontinued & $22(1.0)$ & $40(0.4)$ \\
\hline Initiated & $1036(44.9)$ & $2770(27.1)$ \\
\hline Initiated and discontinued & $339(14.7)$ & 369 (3.6) \\
\hline \multicolumn{3}{|l|}{ Beta-blocker (\%) } \\
\hline Continued & $75(3.2)$ & $419(4.1)$ \\
\hline Discontinued & $20(0.9)$ & $23(0.2)$ \\
\hline Initiated & $873(37.8)$ & $2611(25.5)$ \\
\hline Initiated and discontinued & $549(23.8)$ & $525(5.1)$ \\
\hline \multicolumn{3}{|l|}{ Calcium-channel blocker (\%) } \\
\hline Continued & $36(1.6)$ & $135(1.3)$ \\
\hline Discontinued & $<10(<0.4)$ & $13(0.1)$ \\
\hline Initiated & $287(12.4)$ & $1311(12.8)$ \\
\hline Initiated and discontinued & $81(3.5)$ & $213(2.1)$ \\
\hline \multicolumn{3}{|l|}{ Nitrates (\%) } \\
\hline Continued & $18(0.8)$ & $92(0.9)$ \\
\hline Discontinued & $<10(<0.4)$ & $<10(<0.1)$ \\
\hline Initiated & $502(21.8)$ & $1791(17.5)$ \\
\hline Initiated and discontinued & $165(7.1)$ & $260(2.5)$ \\
\hline \multicolumn{3}{|l|}{ Statins (\%) } \\
\hline Continued & $71(3.1)$ & $332(3.2)$ \\
\hline Discontinued & $<10(<0.4)$ & $<10(<0.1)$ \\
\hline Initiated & 1125 (48.7) & $3102(30.3)$ \\
\hline Initiated and discontinued & $84(3.6)$ & $141(1.4)$ \\
\hline
\end{tabular}

Medication use in selected patients for the CT-first strategy and patients without a CT-first strategy. Continued medication is defined as medication that was started before the chest pain consult and continued for at least 120 following days. Discontinued medication is medication started before the consult and stopped within 120 days. Initiated medication was started within the time frame from chest pain consult until 120 days after the consult. Initiated and discontinued medication was medication that was started and discontinued within 120 days following the chest pain consult.

\section{Subgroup and sensitivity analyses}

The association between having a cardiac CT for diagnostic evaluation of chest pain and all-cause mortality was further investigated in clinically relevant subgroups (figure 3). Men showed greater benefit from a CT-first strategy with respect to all-cause mortality, although $\mathrm{p}$ value for interaction was not significant. This $\mathrm{p}$-value was only significant for type of chest pain ( $\mathrm{p}$-value: 0.013 ) and risk of CAD (p-value: 0.0046), implying that patients with a possible anginal origin of chest pain and with an intermediate to high risk of CAD benefit from a CT-first strategy.

Sensitivity analyses showed that HRs were similar for the different regions of the Netherlands and for the inclusion year of patients (online supplemental table 1). Residence and inclusion year were taken into account during the PS calculation. This suggests a similar distribution of these variables in the CT-first and without CT-first group. Sensitivity analysis of removal of patients $(n=4883)$ from one centre that did not structurally perform cardiac CT including calcium scoring and CCTA showed comparable HR for all-cause mortality $(0.44,95 \% \mathrm{CI}$ 0.28 to 0.67 vs $0.51,95 \%$ CI 0.37 to 0.70 ) and HR for cardiovascular mortality $(0.70,95 \% \mathrm{CI} 0.37$ to 1.33 vs $0.7395 \% \mathrm{CI}$ 0.43 to 1.23 ) to the main matched sample. After removal of all patients with CAG within 10 weeks after their initial visit $(\mathrm{n}=959)$, the HR for all-cause mortality $(0.54,95 \% \mathrm{CI} 0.39$ to $0.74)$ and cardiovascular mortality $(0.78,95 \%$ CI 0.45 to 1.34$)$ changed but within CIs.

\section{DISCUSSION}

The analysis performed in this study demonstrated that upstream inclusion of cardiac CT in diagnostic work-up of patients with chest pain was associated with a significant lowering of all-cause mortality, irrespective of the outcome of the cardiac CT. We also observed a lower cardiovascular mortality rate in CT-first patients, although this difference was not statistically significant. This can be due to the low numbers of a cardiovascular cause of deaths and insufficient registration of the cause of death. Our data also show that patients with a CT-first strategy had more downstream therapeutics and diagnostic testing. This is in accordance with findings from other registries. ${ }^{10}$ The presented results underscore and complement the prominent role of cardiac CT in the diagnostic work-up of patients presenting with chest pain in clinical guidelines, ${ }^{12}$ which has also been internationally addressed. ${ }^{25}$

Based on previous publications, it is assumed that patients that undergo cardiac CT in addition to regular care receive an accurate diagnosis earlier and more often, ${ }^{26}$ after which targeted antianginal and preventative therapy is initiated, resulting in overall superior survival in these patients. ${ }^{27} 28$ The prescription of preventative medication, especially statins, aspirin and beta

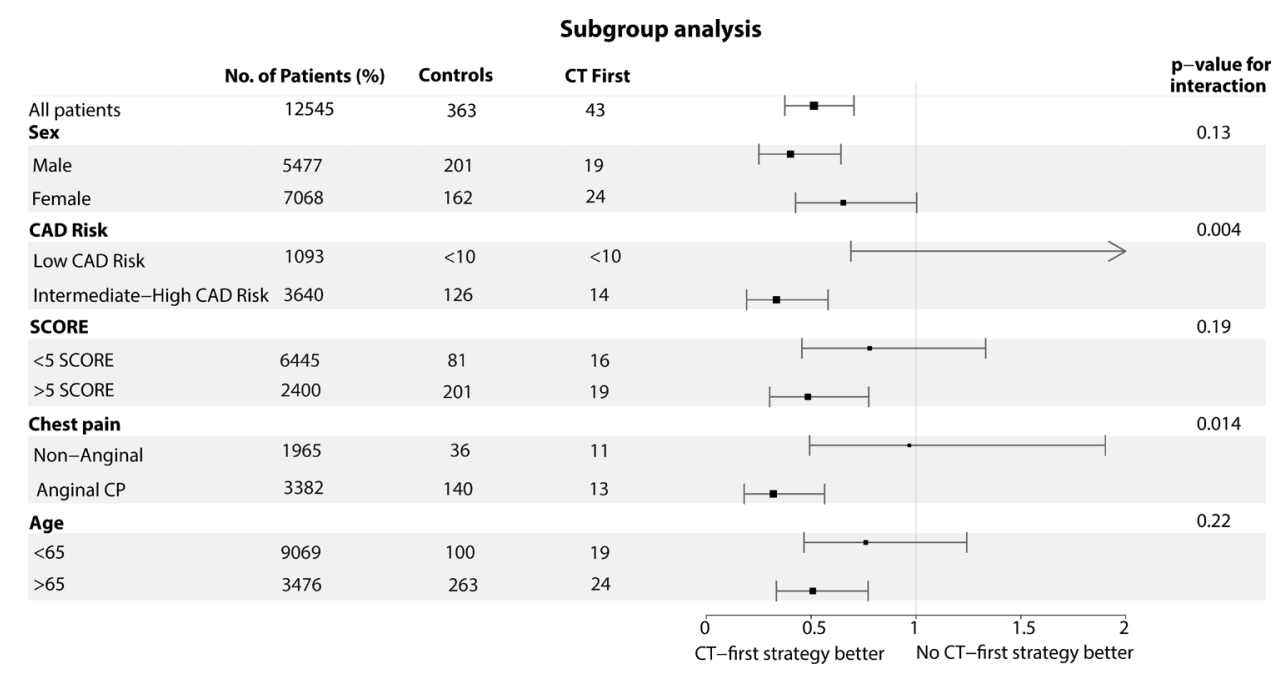

Figure 3 Forest plot of the subgroup analysis. CAD risk is defined as the pretest probability of CAD according to the ESC guidelines of 2019: Low : $<5 \%$, intermediate to high: $>5 \%$. Anginal chest pain includes typical and atypical chest pain. SCORE is the cardiovascular risk score as defined by the Systematic COronary Risk Evaluation. CAD, coronary artery disease; ESC, European Society of Cardiology. 
blockers to patients with CT-verified atherosclerotic lesions was thought to be the major reason for lower mortality rates observed in patients undergoing cardiac CT in the SCOTHEART trial. However, cardiovascular mortality rates were not significantly lower in the CT-first population. One reason for the lower risk of all-cause mortality could be the identification of relevant extracardiac findings, which occur in approximately $3 \%$. Of these, pulmonary nodules make up the largest share $(1.3 \%) .{ }^{29}$ Also the inclusion of patients at lower risk for CAD in the CT-first group that have not been properly identified due to missing values might account for the discrepancy in all-cause and cardiovascular mortality after a CT-first strategy. Finally, the number of events and follow-up time might be too low to establish the effect of lifestyle changes as a result of the visualisation of coronaries on CT. Yet, as we did not include an in-depth analysis of the results of cardiac CT, we could not relate this to the presence of $\mathrm{CAD}$ or atherosclerotic lesions.

In accordance with the SCOT-HEART trial, we observed a higher referral rate for follow-up diagnostics and interventions in CT-first patients. ${ }^{57}$ We did not perform analyses of these downstream costs. Therefore, no statements can be given about the cost-to-benefit ratio in patients with and without a cardiac CT-first strategy. Yet, in the light of a faster and more often correct diagnosis, ${ }^{27}$ lower cumulative diagnostic expenses could be expected for patients with a cardiac CT. ${ }^{26}$ Nevertheless, costeffectiveness of the CT-first strategy needs to be established and should take into account the higher number of revascularisations and medication prescriptions in CT-first patients. ${ }^{10} 30$ On the contrary, the relatively low cost and wide availability of CT makes it accessible for more patients compared with other cardiac imaging strategies.

\section{Strengths and limitations}

The presented study has multiple strengths. First, the use of EHR data aids to address concerns of health volunteer bias and under-representation of patient subgroups that are mostly seen in clinical trials. This is represented in the large study population and equal distribution of sex within the population. Thus, the included population is an actual representation of the population that the guidelines are intended for. Therefore, the results have high external validity. Second, the longer follow-up in this study has incremental value compared with the Danish nationwide registry ${ }^{10}$ and the PROMISE trial ${ }^{8}$ that presented data with a follow-up of respectively 3.6 years and 25 months.

The study was limited by the lack of information on cardiovascular events, including cardiovascular hospitalisations, which hampered the analysis of these events. Another limitation of regular care data is confounding by indication; patients with a higher pretest probability of CAD are more likely to be referred for cardiac CT than patients with a lower pretest probability. To avoid this potential bias, PS matching was used to ensure comparability between patients with and without a CT-first strategy. Regardless, one would have expected that patients with a higher pretest likelihood would show lower survival. Yet, we observed the opposite, vindicating that baseline differences between both groups were properly accounted for. Nonetheless, it is impossible to eliminate or to take into account the influence of unmeasured confounders or instrumental variables in the presented methodology.

To conclude, this study was the first to demonstrate a significantly lower HR for all-cause mortality in patients with chest pain who had a CT-first strategy compared with patients who did not have a CT-first strategy. It is hypothesised that patients with a CT-first strategy obtain a more tailored therapy, including risk-reducing medication. These results support a CT-first strategy for patients with chest pain and strengthen the prominent role for cardiac CT as the primary method for diagnostic work-up of patients with chest pain, as suggested by the current ESC guidelines.

Key messages

What is already known on this subject?

- Upstream incorporation of cardiac CT in the diagnostic pathway (CT-first strategy) has shown to reduce fatal and non-fatal myocardial infarction with $50 \%$ in patients with chest pain in the SCOT-HEART study. Although registry studies and the PROMISE trial could not confirm these findings, international guidelines have incorporated a CT-first strategy in the evaluation of patients with chest pain. Regular care data can aid in building the evidence for this guideline implementation.

\section{What might this study add?}

- A CT-first strategy in patients with chest pain is associated with a lower risk of all-cause mortality (HR: $0.51,95 \% \mathrm{Cl}$ 0.37 to 0.70 ), but not cardiovascular mortality (HR: 0.73 , $95 \% \mathrm{Cl} 0.43$ to 1.24$)$. In patients with a CT-first strategy, prescriptions of risk-reducing and antianginal medication were higher compared with patients without a CT-first strategy.

- Propensity score matching and multiple imputation can reduce bias present in regular care data. These methods enable researchers to use the potential of regular care data to strengthen the evidence base for clinical practices.

\section{How might this impact on clinical practice?}

- Patients undergoing a cardiac CT as part of their diagnostic work-up for chest pain might have increased survival, compared with patients undergoing usual care. It is hypothesised this is due to more timely and accurate diagnosis of the origin of chest pain, resulting in targeted therapeutics and initiation of antianginal and preventative medication.

Twitter Hester M den Ruijter @InnovatieHester

Acknowledgements Results are based on calculations by the authors using nonpublic microdata from Statistics Netherlands.

Contributors KRS, NCOM, HMdR and LH designed the study. KRS cleaned the raw data, performed data analyses and wrote the manuscript. NCOM and HMdR supported data analysis and critically reviewed the manuscript. IIT, LH and GAS collected the raw data and reviewed the manuscript. YA, PvdH and JN critically reviewed the manuscript. LH is responsible for the overall contact and acts as the guarantor.

Funding This study was funded by the Dutch Heart Foundation (CVON-Al: 2018B017).

Competing interests LH, IIT and GAS are employed by Cardiology Centres of the Netherlands.

Patient consent for publication Not applicable.

Provenance and peer review Not commissioned; externally peer reviewed.

Data availability statement Data are available on reasonable request. The CCN data is not publicly available due to ethical and data protection constraints, but are available from the corresponding author on reasonable request. The results of presented survival analyses are based on calculations by the authors using non-public microdata from Statistics Netherlands. Under certain conditions, these microdata are accessible for statistical and scientific research. For further information: microdata@cbs.nl. 
Supplemental material This content has been supplied by the author(s). It has not been vetted by BMJ Publishing Group Limited (BMJ) and may not have been peer-reviewed. Any opinions or recommendations discussed are solely those of the author(s) and are not endorsed by BMJ. BMJ disclaims all liability and responsibility arising from any reliance placed on the content. Where the content includes any translated material, BMJ does not warrant the accuracy and reliability of the translations (including but not limited to local regulations, clinical guidelines, terminology, drug names and drug dosages), and is not responsible for any error and/or omissions arising from translation and adaptation or otherwise.

Open access This is an open access article distributed in accordance with the Creative Commons Attribution Non Commercial (CC BY-NC 4.0) license, which permits others to distribute, remix, adapt, build upon this work non-commercially, and license their derivative works on different terms, provided the original work is properly cited, appropriate credit is given, any changes made indicated, and the use is non-commercial. See: http://creativecommons.org/licenses/by-nc/4.0/.

ORCID iDs

Klaske R Siegersma http://orcid.org/0000-0001-6377-6867

Hester M den Ruijter http://orcid.org/0000-0001-9762-014X

\section{REFERENCES}

1 Arad Y, Spadaro LA, Roth M, et al. Treatment of asymptomatic adults with elevated coronary calcium scores with atorvastatin, vitamin C, and vitamin E: the St. Francis heart study randomized clinical trial. J Am Coll Cardiol 2005;46:166-72.

2 Budoff MJ, Shaw LJ, Liu ST, et al. Long-Term prognosis associated with coronary calcification: observations from a Registry of 25,253 patients. J Am Coll Cardiol 2007:49:1860-70

3 Detrano R, Guerci AD, Carr JJ, et al. Coronary calcium as a predictor of coronary events in four racial or ethnic groups. N Engl J Med 2008;358:1336-45.

4 Yeboah J, McClelland RL, Polonsky TS, et al. Comparison of novel risk markers for improvement in cardiovascular risk assessment in intermediate-risk individuals. JAMA 2012;308:788-95

5 SCOT-HEART Investigators, Newby DE, Adamson PD, et al. Coronary CT angiography and 5-year risk of myocardial infarction. N Eng/ J Med 2018;379:924-33.

6 Villines TC, Hulten EA, Shaw $L$, et al. Prevalence and severity of coronary artery disease and adverse events among symptomatic patients with coronary artery calcification scores of zero undergoing coronary computed tomography angiography: results from the CONFIRM (coronary CT angiography evaluation for clinical outcomes: an international multicenter) registry. J Am Coll Cardiol 2011;58:2533-40.

7 Neglia D, Rovai D, Caselli C, et al. Detection of significant coronary artery disease by noninvasive anatomical and functional imaging. Circ Cardiovasc Imaging 2015:8:1-10

8 Douglas PS, Hoffmann U, Patel MR, et al. Outcomes of anatomical versus functional testing for coronary artery disease. N Eng/ I Med 2015;372:1291-300.

9 National Institute for Health and Clinical Excellence. Recent-onset chest pain of suspected cardiac origin: assessment and diagnosis. Clin Guidel [CG95] 2010.

10 Jørgensen ME, Andersson C, Nørgaard BL, et al. Functional Testing or Coronary Computed Tomography Angiography in Patients With Stable Coronary Artery Disease. J Am Coll Cardiol 2017;69:1761-70.
11 Roifman I, Wijeysundera HC, Austin PC, et al. Comparison of anatomic and clinical outcomes in patients undergoing alternative initial noninvasive testing strategies for the diagnosis of stable coronary artery disease. J Am Heart Assoc 2017;6:1-13.

12 Knuuti J, Wijns W, Saraste A, et al. 2019 ESC guidelines for the diagnosis and management of chronic coronary syndromes. Eur Heart J 2020;41:407-77.

13 Pinsky PF, Miller A, Kramer BS, et al. Evidence of a healthy volunteer effect in the prostate, lung, colorectal, and ovarian cancer screening trial. Am J Epidemiol 2007;165:874-81.

14 Leening MJG, Heeringa J, Deckers JW, et al. Healthy volunteer effect and cardiovascular risk. Epidemiology 2014;25:470-1.

15 Pilote L, Raparelli V. Participation of women in clinical trials: not yet time to rest on our laurels. J Am Coll Cardiol 2018;71:1970-2.

16 Sardar MR, Badri M, Prince CT, et al. Underrepresentation of women, elderly patients, and racial minorities in the randomized trials used for cardiovascular guidelines. JAMA Intern Med 2014;174:1868-70.

17 Van Spall HGC, Toren A, Kiss A, et al. Eligibility criteria of randomized controlled trials published in high-impact general medical journals: a systematic sampling review. JAMA 2007;297:1233-40.

18 Bots SH, Siegersma KR, Onland-Moret NC, et al. Routine clinical care data from thirteen cardiac outpatient clinics: design of the cardiology centers of the Netherlands (CCN) database. BMC Cardiovasc Disord 2021;21:1-9.

19 Diamond GA. A clinically relevant classification of chest discomfort. J Am Coll Cardiol 1983;1:574-5.

20 Buuren Svan, Groothuis-Oudshoorn K. mice : Multivariate Imputation by Chained Equations in R. J Stat Softw 2011;45:1-67.

21 Austin PC. An introduction to propensity score methods for reducing the effects of confounding in observational studies. Multivariate Behav Res 2011;46:399-424.

22 DE H, Imai K, King G. Matchlt: nonparametric preprocessing for parametric causal inference. J Stat Softw 2011;42:1-28.

23 Conroy RM, Pyörälä K, Fitzgerald AP, et al. Estimation of ten-year risk of fatal cardiovascular disease in Europe: the SCORE project. Eur Heart J 2003;24:987-1003.

24 Juarez-Orozco LE, Saraste A, Capodanno D, et al. Impact of a decreasing pre-test probability on the performance of diagnostic tests for coronary artery disease. Eur Heart J Cardiovasc Imaging 2019;20:1198-207.

25 Poon M, Lesser JR, Biga C, et al. Current evidence and recommendations for coronary cta first in evaluation of stable coronary artery disease. J Am Coll Cardiol 2020;76:1358-62.

26 Lubbers M, Dedic A, Coenen A, et al. Calcium imaging and selective computed tomography angiography in comparison to functional testing for suspected coronary artery disease: the multicentre, randomized crescent trial. Eur Heart J 2016;37:1232-43.

27 Williams MC, Hunter A, Shah ASV, et al. Use of coronary computed tomographic angiography to guide management of patients with coronary disease. J Am Coll Cardiol 2016;67:1759-68.

28 SCOT-HEART investigators. CT coronary angiography in patients with suspected angina due to coronary heart disease (SCOT-HEART): an open-label, parallel-group, multicentre trial. Lancet 2015;385:2383-91.

29 Karius P, Lembcke A, Sokolowski FC, et al. Extracardiac findings on coronary computed tomography angiography in patients without significant coronary artery disease. Eur Radiol 2019;29:1714-23.

30 Zeb I, Abbas N, Nasir K, et al. Coronary computed tomography as a cost-effective test strategy for coronary artery disease assessment - a systematic review. Atherosclerosis 2014;234:426-35. 